\title{
Evanescent wave optical binding forces on spherical microparticles
}

\author{
XIANG HAN ${ }^{1,2}$ AND PhILIP H. JONES ${ }^{2, *}$ \\ ${ }^{1}$ College of Opto-Electric Science and Engineering, National University of Defense Technology, Changsha, China, 410073 \\ ${ }^{2}$ Department of Physics and Astronomy, University College London, Gower Street, London WC1E 6BT, United Kingdom \\ ${ }^{*}$ Corresponding author: philip.jones@ucl.ac.uk
}

Compiled July 1, 2015

In this paper we demonstrate stable optical binding of spherical microparticles in counter-propagating evanescent optical fields formed by total reflection at a dielectric interface. The microspheres are observed to form one-dimensional chains oriented parallel to the direction of propagation of the beams. We characterize the strength of the optical binding interaction by measuring the extent of Brownian position fluctuations of the optically bound microspheres and relating this to a binding spring constant acting between adjacent particles. A stronger binding interaction is observed for particles near the middle of the chain, and the dependence of the binding strength on incident laser power and number of particles in the chain is determined.

(C) 2015 Optical Society of America

OCIS codes: (140.7010) Laser trapping; (170.4520) Optical confinement and manipulation; (350.4855) Optical tweezers or optical manipulation.

http://dx.doi.org/10.1364/ol.XX.XXXXXX

\section{INTRODUCTION}

Optical binding [1-3] refers to the spontaneous arrangement of microscopic particles in an intense optical field as a result of multiple scattering between the particles. Although the ability of light to exert a significant force on matter has been widely exploited in the case of manipulating single particles in optical tweezers [4], the phenomenon of optical binding has been much less studied. One often-used experimental geometry is to use counter-propagating beams [5-7], where one-dimensional chains of longitudinally optically bound microscopic particles form where the beams overlap. Optical binding has also been observed with gold [8] and silver [9] nanoparticles, and carbon nanotube bundles [10] in a number of different experimental configurations. Very recent work by Brügger et al. [11] has demonstrated the possibility of controlling hte forces arising rom optical scattering interactions between pairs of colloidal particles using a random light field.

Evanescent fields are formed at the interface between dielectric media with different refractive indices when an electromagnetic wave undergoes total internal reflection. In the evanescent wave power is transmitted parallel to the interface. In 1992 this was exploited by Kawata and Sugiura [12] who showed that electromagnetic momentum could be transferred to microscopic particles immersed in the evanescent field, resulting in propulsion of the particles parallel to the direction of propagation. Garcés-Chávez et al [13] showed that by balancing the radiation pressure from the evanescent field using counter-propagating waves, particles could be stably trapped and also form optically bound ordered structures of large numbers of particles over an extended area. Subsequently Mellor and Bain [14] observed a variety of two-dimensional structures in evanescent wave optical binding that were a function of particle size and laser polarization. An alternative means of generating an evanescent field for optical binding is to use an optical fiber tapered to a sub-micron waist [15], which can either propel particles if the field propagates in one direction [16], or bind stable chains [17] with counter-propagating beams. Evanescent wave propulsion has found recently application in the separation of metallic particles based on their differing plasmonic response to two-color optical fields [18].

In this work we study the optical binding interactions in one-dimensional chains of microscopic spherical particles that form in the evanescent field above a planar dielectric interface. We consider the optically-mediated interactions between particles to be analogous to linear springs, and characterize the optical spring constants by measuring the Brownian motion of the bound particles. By doing so we are able to measure the variation in spring constant along the length of the chain, and also to determine the dependence of the optical binding interaction strength (as characterized by the average spring constant) on laser power and the number of particles in the chain.

\section{EXPERIMENTAL APPARATUS}

The experimental apparatus is shown in Fig. 1. The beam from a Nd:YAG laser, wavelength $\lambda=1064 \mathrm{~nm}$ and maximum output power $P_{\max }=2 \mathrm{~W}$, is expanded by a telescope and focused by a lens (focal length $F_{1}=150 \mathrm{~mm}$ ) to a waist measuring ap- 


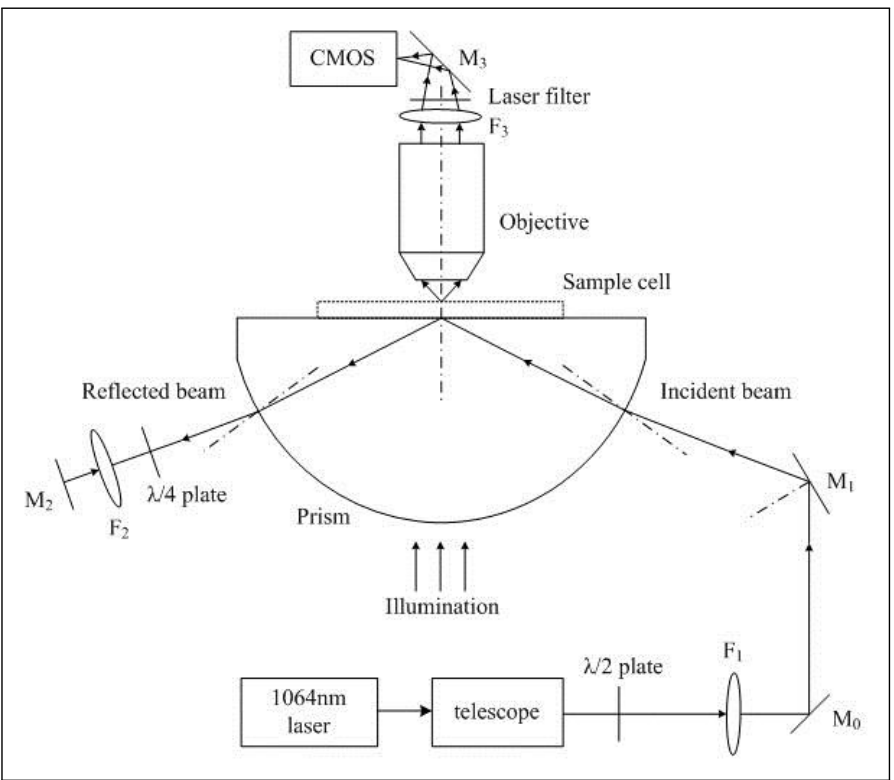

Fig. 1. Experimental apparatus. The expanded beam of the laser is loosely focused by lens with focal length $F_{1}$ onto the planar surface of the near-hemispherical prism at the critical angle for the glass-water interface. The lens with focal length $F_{2}$ collimates then re-focuses the retro-reflected beam. Samples are observed by imaging onto the CMOS camera using a the $\times 100$ objective lens and tube lens, $F_{3}$.

proximately $70 \mu \mathrm{m} \times 50 \mu \mathrm{m}$ at the planar surface of a near hemispherical plano-convex lens. A half-wave plate is used to set the direction of linear polarization to be perpendicular to the planar surface, and the weakly focused beam is incident on the surface at an angle slightly greater than the critical angle for a glass-water interface. The beam undergoes total internal reflection at this interface, then is collimated (by a lens of focal length $F_{2}=150 \mathrm{~mm}$ ), retro-reflected and re-focused to a waist of approximately the same dimensions which overlaps the waist of the first incident beam. A quarter-wave plate is used to set the polarization of the reflected beam to be orthogonal to the incident beam to avoid the formation of interference fringes in the evanescent field.

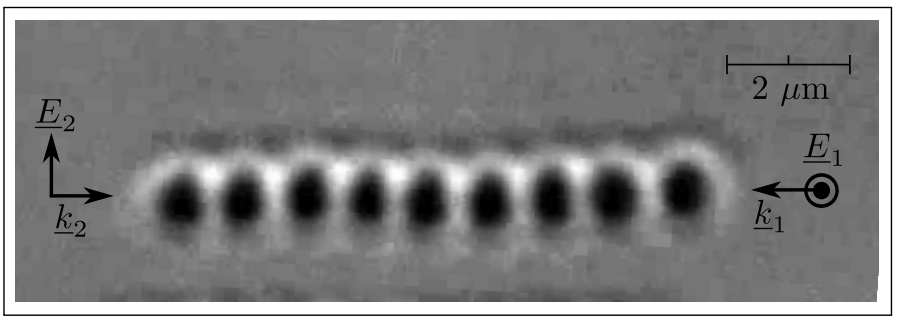

Fig. 2. An optically bound chain of nine $1 \mu \mathrm{m}$ diameter silica microspheres which forms spontaneously in the counterpropagating evanescent fields. The wavevectors $\left(\underline{k}_{1}, \underline{k}_{2}\right)$ and electric field polarizations $\left(\underline{E}_{1}, \underline{E}_{2}\right)$ are oriented as shown.

Samples are made by diluting $0.5 \mu \mathrm{l}$ of $1 \mu \mathrm{m}$ diameter silica microspheres solution (initial concentration $50 \mathrm{mg} / \mathrm{ml}$ ) with $50 \mu \mathrm{l}$ of deionised water containing $10 \%$ of the surfactant Triton$X$ to prevent the microspheres from irreversibly sticking together or to the plane surface. A cell is made on the lens surface using an adhesive SecureSeal spacer (diameter $9 \mathrm{~mm}$, depth $0.12 \mathrm{~mm}$ ), filled with diluted bead solution and sealed with a No. 1.5 cover slip. The sample is imaged through a $\times 100 \mathrm{ob}-$ jective and a tube lens (focal length $F_{3}=175 \mathrm{~mm}$ ) onto a 1.3 megapixel CMOS camera, and a colored glass filter is used to eliminate scattered infra-red laser light.
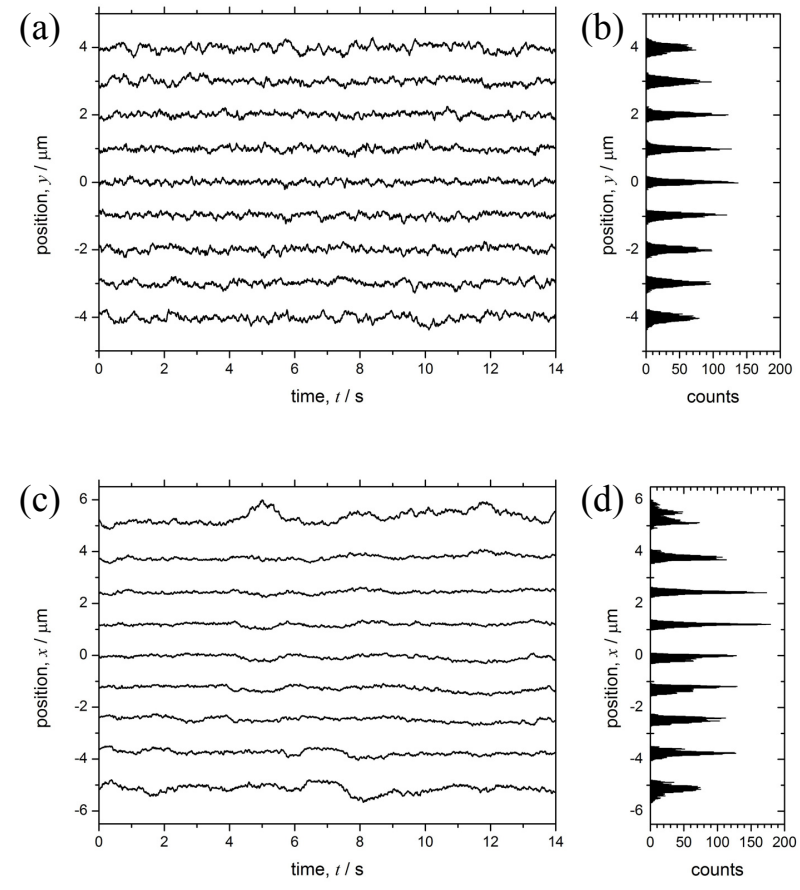

Fig. 3. (a) Particle position fluctuations relative to the centerof-mass in the direction perpendicular to the chain axis; (b) Histograms of particle position fluctuations. In both cases the data have been separated for clarity such that the average positions of adjacent particles differ by $1 \mu \mathrm{m}$; (c) Particle position fluctuations relative to the center-of-mass in the direction parallel to the chain axis; (d) Histograms of particle position fluctuations. The power in a single incident beam was $P_{1}=324 \mathrm{~mW}$.

For incident laser powers of a few hundred milliwatts the microspheres are observed to spontaneously form optically bound chains parallel to the directions of propagation of the laser beams. In our apparatus neither the location of chain formation nor the number of particles in the chain are actively controlled [19]. An example of one such chain formed of $N=9$ particles is shown in Fig. 2. For the range of laser powers used the optical binding interaction between the particles is sufficiently weak that Brownian fluctuations in the relative positions of the particles are readily observable in video recordings. The motion of the particles in two dimensions (i.e. parallel to the planar surface) was recorded by digital video microscopy, typically for several thousand frames, and subject to particle tracking [20] to extract the individual particle trajectories $\left\{x_{i}(t), y_{i}(t), i=1 \ldots N\right\}$. An example of the Brownian motion of the nine particles in the chain depicted in Fig 2 recorded over $14 \mathrm{~s}$ is shown in Fig. 3. Fluctuations in the direction perpendicular to the chain axis are plotted in Fig. 3 after subtraction of the relatively slowly fluctuating center-of-mass motion of the chain, i.e. $y_{i}-y_{0}, i=1 \ldots 9$, 
where $y_{0}=\frac{1}{N} \sum_{i=1}^{N} y_{i}$, (since there is only very weak transverse confinement of the chain from the gradient of intensity in the background gaussian laser beams). Histograms of the fluctuations of the particle positions relative to the center-of-mass are shown in Fig. 3(b). Note that the particle trajectories and the histograms (which are all centered at $\left\langle y_{i}\right\rangle=0$ ) have been separated for clarity of display. Fluctuations in particle position relative to the center-of-mass in the direction parallel to the chain axis $\left(x_{i}-x_{0}, \quad i=1 \ldots 9\right.$ and $\left.x_{0}=\frac{1}{N} \sum_{i=1}^{N} x_{i}\right)$ are shown in Fig. 3(c), and histograms in Fig. 3(d). As might be expected, particles at the ends of the chains exhibit a larger variance in position fluctuations (relative to the center-of-mass) in both dimensions than particles near the middle. As previously found in calculations and experiments for longitudinal optical binding with Bessel beams [21] we also observe an approximately symmetric variation in average particle separation along the chain from $1.2 \mu \mathrm{m}$ near the middle, to $1.4 \mu \mathrm{m}$ at the ends due to the increased effects of forward-scattered light on the end particles from all other particles in the chain.

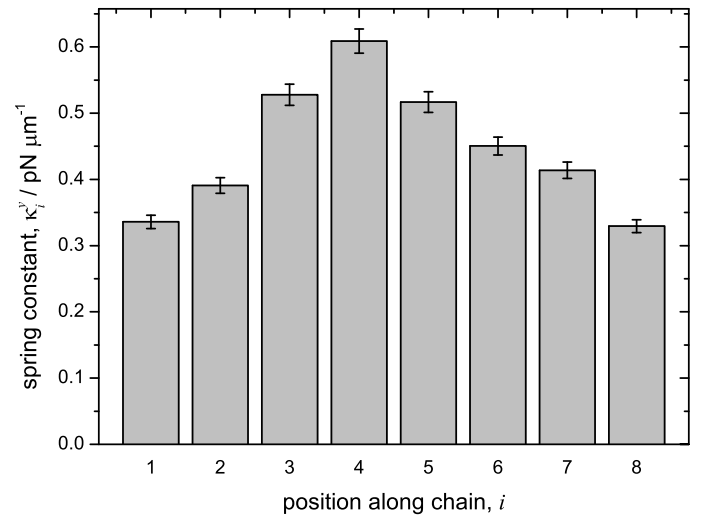

Fig. 4. Variation of the optical spring constant, $\kappa_{i}^{y}$, between particles $i$ and $i+1$ measured in a chain of $N=9$ particles for incident power in a single beam $P_{1}=324 \mathrm{~mW}$.

\section{RESULTS AND ANALYSIS}

We model the optically bound chain as a system of $N$ particles connected by $N-1$ linear springs, that is each particle is confined in a potential well that is harmonic with the separation between adjacent particles [8]. The stiffness of the spring joining particles $i$ and $i+1$ is denoted $\kappa_{i}$. For the remainder of this paper we will use the motion perpendicular to the chain axis to characterize the optical interactions. We therefore consider the optical restoring force acting between the pair of particles $i$ and $i+1$ to be:

$$
F_{i}^{y}=-\kappa_{i}^{y}\left(y_{i+1}-y_{i}\right) .
$$

By equipartition of energy, the stiffness (spring constant) of the bond between the particles can be found from the variance of the fluctuations in their relative separation, i.e.

$$
\kappa_{i}^{y} \operatorname{var}\left(y_{i+1}-y_{i}\right)=k_{\mathrm{B}} T .
$$

The advantage of the equipartition method for determining the optical spring constant over other methods that are commonly

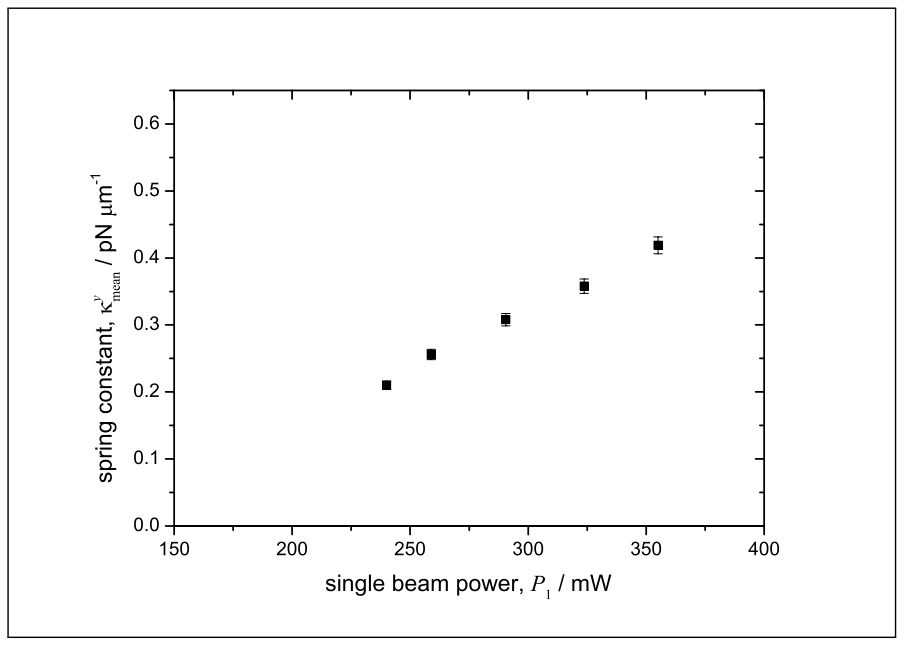

Fig. 5. The averaged spring constant, $\kappa_{\text {mean, }}$ as a function of laser power, $P_{1}$, for a chain containing $N=9$ particles. The average spring constant increases linearly with laser power above a threshold power for stable chain formation.

used in, for example, calibration of optical tweezers experiments [22] is that this method does not require consideration of the complex hydrodynamic coupling between the optically bound spheres and between the spheres and the adjacent plane surface [23].

An example of the measured spring constants $\kappa_{i}^{y}$ as a function of position along the length of the chain is shown in Fig. 4 for the $N=9$ particle chain. Evident here is the non-uniformity of the optical binding interaction along the chain. A simple model for understanding optical binding describes the interaction between each pair of particles in a chain as arising from the field scattered by one onto the other, with an amplitude that varies as inversely proportional to their separation [24]. The total field incident on particle $i$ is thus the sum of the incident field (propagating in $+x$ and $-x$ directions) and the fields scattered by the the other particles in the chain, from $1 \ldots(i-1)$ on one side, and from $(i+1) \ldots N$ on the other. The resultant intensity at the location of each particle would therefore be expected to vary along the length of the chain to produce a binding interaction that is strongest in the center as observed here, although an exact calculation is rendered more complicated due to the influence of scattering by the adjacent surface [25].

We have made a systematic investigation of the strength of the optical binding interaction for chains formed with up to $N=12$ particles and a range of laser beam powers up to $P_{1}=355 \mathrm{~mW}$. In order to compare chains of different lengths bound at different laser powers we use the value of the spring constant averaged over the whole chain $\kappa_{\text {mean }}^{y}$. Particle trajectories obtained from video recordings of up to 5000 frames (depending on the stability of the chain) were analyzed for various combinations of particle number and laser power. The most stable chains, i.e. those that persisted longest against thermal fluctuations, were those with larger numbers of particles and higher laser power, although the use of higher power in particular tended to draw additional particles into the chain in an uncontrolled manner. At very low laser powers chains continually broke and re-formed as a result of thermal fluctuations and it was not possible to quantify the binding interaction. An example of the measured average spring constant, $\kappa_{\text {mean, as a }}^{y}$ 


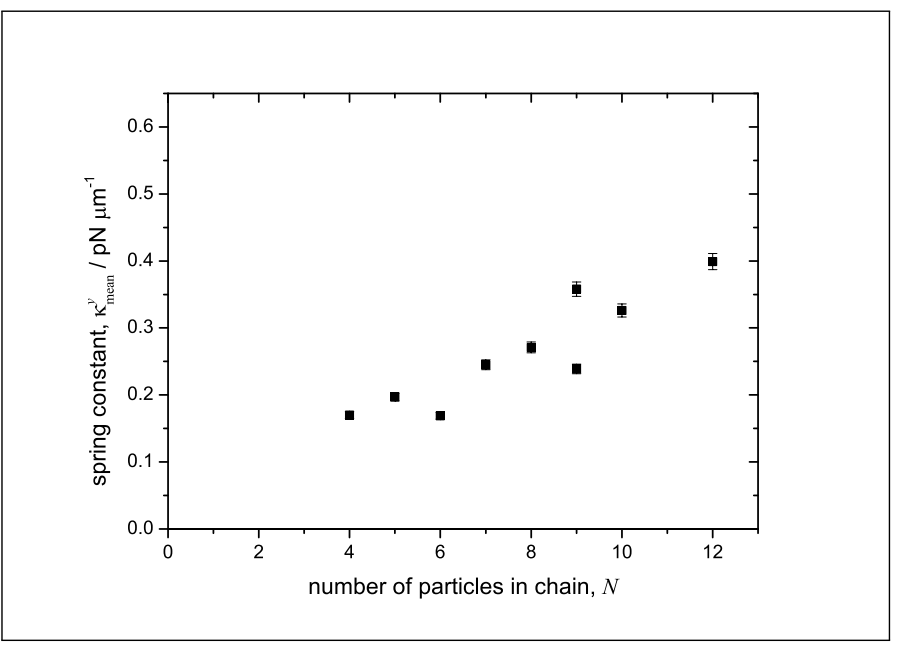

Fig. 6. The averaged spring constant, $\kappa_{\text {mean, }}$ as a function of the number of particles, $N$, in the chain measured at constant laser power, $P_{1}=324 \mathrm{~mW}$. Within this range the averaged spring constant increases linearly with the number of particles in the chain.

function of laser power for a constant number of particles is shown in Fig. 5. For the range of laser powers investigated where stable chains formed for long enough to take measurements, the average spring constant can be seen to increase linear with laser power above a threshold value. This can be interpreted as a minimum power required for stable chain formation. A linear fit to the data reveals the threshold for this chain to be $P_{\text {thr }}=120 \pm 10 \mathrm{~mW}$.

The dependence of the optical binding spring constant on particle number, $N$, is elaborated in Fig. 6, where the stiffness is measured as a function of particle number for fixed laser power. As recognized in [24] the first order scattering solution, which predicts the optical binding force to increase with the logarithm of the particle number, underestimates the rate of growth of optical binding interaction with particle number. For the range of chain lengths investigated, we find that the stiffness increases with the number of particles. Even at the longest chains found in our experiment $(N=12)$ we have not been able to observe any saturation in the optical binding force. The limitation on chain length in these experiments arises only from the requirement that the chains self-assemble due to optical binding in a relatively uncluttered environment free from hydrodynamic interactions with unbound particles, meaning that low densities of particles must be used. Controlled assembly [19] using an additional optical tweezers would permit the regime of much larger particle numbers to be investigated to locate any roll-off in the optical binding force with increasing length.

\section{CONCLUSION}

We have studied here the strength of interaction in longitudinal optical binding of spherical microparticles in an evanescent wave trap. The interaction strength is characterized by an optical spring constant between adjacent particles in a onedimensional chain obtained by analysis of their relative Brownian motion. We find that the optical binding interaction strength scales linearly with the binding laser beam power once a threshold for optical binding to occur is reached, and that it also scales linearly with the number of particles in the chain.

\section{FUNDING INFORMATION}

This work is supported by the Leverhulme Trust. Xiang Han thanks the China Scholarship Council for a visiting studentship.

\section{REFERENCES}

1. M. M. Burns, J.-M. Fournier, and J. A. Golovchenko, Phys. Rev. Lett. 63, 1233-1236 (1989).

2. M. M. Burns, J.-M. Fournier, and J. A. Golovchenko, Science 249, 749-754 (1990).

3. K. Dholakia and P. Zemánek, Rev. Mod. Phys. 82, 1767 (2010).

4. A. Ashkin, J. M. Dziedzic, J. E. Bjorkholm, and S. Chu, Opt. Lett. 11, 288-290 (1986).

5. S. A. Tatarkova, A. E. Carruthers, and K. Dholakia, Phys. Rev. Lett. 89, 283901 (2002).

6. W. Singer, M. Frick, S. Bernet, and M. Ritsch-Marte, J. Opt. Soc. Am. B 20, 1568-1574 (2003).

7. R. Gordon, M. Kawano, J. T. Blakely, and D. Sinton, Phys. Rev. B 77, 245125 (2008).

8. V. Demergis and E.-L. Florin, Nano Lett. 12, 5756-5760 (2012).

9. Z. Yan, R. A. Shah, G. Chado, S. K. Gray, M. Pelton, and N. F. Scherer, ACS Nano 7, 1790-1802 (2013).

10. O. M. Maragò, P. H. Jones, P. G. Gucciardi, G. Volpe, and A. C. Ferrari, Nat. Nanotechnology 8, 807-819 (2013).

11. G. Brügger, L. S. Froufe-Pérez, F. Scheffold, and J. J. Sáenz, Nat. Commun. 6, 7460 (2015).

12. S. Kawata and T. Sugiura, Opt. Lett. 17, 772-774 (1992).

13. V. Garcés-Chávez, K. Dholakia, and G. C. Spalding, Appl. Phys. Lett. 86, 031106 (2005).

14. C. D. Mellor and C. D. Bain, ChemPhysChem 7, 329-332 (2006).

15. G. Brambilla, G. S. Murugan, J. S. Wilkinson, and D. J. Richardson, Opt. Lett. 32, 3041-3043 (2007).

16. S. E. Skelton, M. Sergides, R. Patel, E. Karczewska, O. M. Marago, and P. H. Jones, J. Quant. Spectrosc. Radiat. Transfer 113, 25122520 (2012).

17. M. C. Frawley, I. Gusachenko, V. G. Truong, M. Sergides, and S. Nic Chormaic, Opt. Express 22, 16322-16334 (2014).

18. M. Ploschner, T. Čižmár, M. Mazilu, A. Di Falco, and K. Dholakia, Nano Lett. 12, 1923-1927 (2012).

19. M. D. Summers, R. D. Dear, J. M. Taylor, and G. A. Ritchie, Opt. Express 20, 1001-1012 (2012).

20. J. C. Crocker and D. G. Grier, J. Colloid Interface Sci. 179, 298-310 (1996).

21. V. Karásek, O. Brzobohatỳ, and P. Zemánek, J. Opt. A: Pure Appl. Opt. 11, 034009 (2009).

22. G. Pesce, G. Volpe, O. M. Marago, P. H. Jones, S. Gigan, A. Sasso, and G. Volpe, J. Opt. Soc. Am. B 32, $84-98$ (2015).

23. M. Sarshar, W. T. Wong, and B. Anvari, J. Biomed. Opt. 19, 115001115001 (2014).

24. J. M. Taylor, L. Y. Wong, C. D. Bain, and G. D. Love, Opt. Express 16, 6921-6929 (2008).

25. P. C. Chaumet and M. Nieto-Vesperinas, Phys. Rev. B 61, 1411914127 (2000). 


\section{INFORMATIONAL FIFTH PAGE}

\section{REFERENCES}

1. M. M. Burns, J.-M. Fournier, and J. A. Golovchenko, "Optical binding," Phys. Rev. Lett. 63, 1233-1236 (1989).

2. M. M. Burns, J.-M. Fournier, and J. A. Golovchenko, "Optical matter: crystallization and binding in intense optical fields," Science 249, 749754 (1990).

3. K. Dholakia and P. Zemánek, "Colloquium: gripped by light: optical binding," Rev. Mod. Phys. 82, 1767 (2010).

4. A. Ashkin, J. M. Dziedzic, J. E. Bjorkholm, and S. Chu, "Observation of a single-beam gradient force optical trap for dielectric particles," Optics Letters 11, 288-290 (1986).

5. S. A. Tatarkova, A. E. Carruthers, and K. Dholakia, "One-dimensional optically bound arrays of microscopic particles," Phys. Rev. Lett. 89, 283901 (2002).

6. W. Singer, M. Frick, S. Bernet, and M. Ritsch-Marte, "Self-organized array of regularly spaced microbeads in a fiber-optical trap," J. Opt. Soc. Am. B 20, 1568-1574 (2003).

7. R. Gordon, M. Kawano, J. T. Blakely, and D. Sinton, "Optohydrodynamic theory of particles in a dual-beam optical trap," Phys. Rev. B 77, 245125 (2008).

8. V. Demergis and E.-L. Florin, "Ultrastrong optical binding of metallic nanoparticles," Nano Letters 12, 5756-5760 (2012).

9. Z. Yan, R. A. Shah, G. Chado, S. K. Gray, M. Pelton, and N. F. Scherer, "Guiding spatial arrangements of silver nanoparticles by optical binding interactions in shaped light fields," ACS Nano 7, 17901802 (2013).

10. O. M. Maragò, P. H. Jones, P. G. Gucciardi, G. Volpe, and A. C. Ferrari, "Optical trapping and manipulation of nanostructures," Nature Nanotechnology 8, 807-819 (2013).

11. G. Brügger, L. S. Froufe-Pérez, F. Scheffold, and J. .J. Sáenz, "Controlling dispersion forces between small particles with artificially created random light fields," Nat. Commun. 6, 7460 (2015).

12. S. Kawata and T. Sugiura, "Movement of micrometer-sized particles in the evanescent field of a laser beam," Opt. Lett. 17, 772-774 (1992).

13. V. Garcés-Chávez, K. Dholakia, and G. C. Spalding, "Extended-area optically induced organization of microparticles on a surface," Applied Physics Letters 86, 031106 (2005).

14. C. D. Mellor and C. D. Bain, "Array formation in evanescent waves," ChemPhysChem 7, 329-332 (2006).

15. G. Brambilla, G. S. Murugan, J. S. Wilkinson, and D. J. Richardson, "Optical manipulation of microspheres along a subwavelength optical wire," Opt. Lett. 32, 3041-3043 (2007).

16. S. E. Skelton, M. Sergides, R. Patel, E. Karczewska, O. M. Marago, and P. H. Jones, "Evanescent wave optical trapping and transport of micro-and nanoparticles on tapered optical fibers," J. Quant. Spectrosc. Radiat. Transfer 113, 2512-2520 (2012).

17. M. C. Frawley, I. Gusachenko, V. G. Truong, M. Sergides, and S. Nic Chormaic, "Selective particle trapping and optical binding in the evanescent field of an optical nanofiber," Opt. Express 22, 1632216334 (2014).

18. M. Ploschner, T. Čižmár, M. Mazilu, A. Di Falco, and K. Dholakia, "Bidirectional optical sorting of gold nanoparticles," Nano Letters 12, 19231927 (2012).

19. M. D. Summers, R. D. Dear, J. M. Taylor, and G. A. Ritchie, "Directed assembly of optically bound matter," Opt. Express 20, 1001-1012 (2012).

20. J. C. Crocker and D. G. Grier, "Methods of digital video microscopy for colloidal studies," J. Colloid Interface Sci. 179, 298-310 (1996).

21. V. Karásek, O. Brzobohatỳ, and P. Zemánek, "Longitudinal optical binding of several spherical particles studied by the coupled dipole method," J. Opt. A: Pure Appl. Opt. 11, 034009 (2009).

22. G. Pesce, G. Volpe, O. M. Marago, P. H. Jones, S. Gigan, A. Sasso, and G. Volpe, "Step-by-step guide to the realization of advanced optical tweezers," J. Opt. Soc. Am. B 32, 84 - 98 (2015).

23. M. Sarshar, W. T. Wong, and B. Anvari, "Comparative study of methods to calibrate the stiffness of a single-beam gradient-force optical tweezers over various laser trapping powers," J. Biomed. Opt. 19, 115001-115001 (2014).

24. J. M. Taylor, L. Y. Wong, C. D. Bain, and G. D. Love, "Emergent properties in optically bound matter," Opt. Express 16, 6921-6929 (2008).

25. P. C. Chaumet and M. Nieto-Vesperinas, "Coupled dipole method determination of the electromagnetic force on a particle over a flat dielectric substrate," Phys. Rev. B 61, 14119-14127 (2000). 